\title{
Design of a Deviation Correction and Positioning Device for Metering Current Transformer Automated Testing Procedure based on Adaptive PLC Control
}

\author{
Meng ZHANG ${ }^{1, a,{ }^{*},}$ Jian YANG $^{1}$ and Yuqi WANG ${ }^{1}$ \\ ${ }^{1}$ State Grid Shandong Electric Power Research Institute, Jinan, Shandong, 250003, P. R. China \\ amengzhangxjtu@126.com
}

Keywords: PLC Control, Position correction, Metering Current Transformer

\begin{abstract}
This paper presents a mechatronic device that finely corrects the fastening position of test standards and applies it to the automated testing procedure of metering current transformer.In the automated test, there is a certain deviation between the measurement transformer size and the standard size, and the actual small deviation will affect the contact area of the secondary crimping, which will affect the consistency and accuracy of the test results. In this paper,we design a corrective pushing component and a positioning clamp assembly structure, and used an adaptive control algorithm to effectively fix and correct the position of the tested transformer. The actual results show that the test position of the tested product is effectively calibrated, and the accuracy and stability of the automated test are greatly improved.
\end{abstract}

\section{Introduction}

Current transformers have been widely used in current monitoring, energy metering and relay protection. In power systems, transformers can be used to electrically isolate high-side and low-side equipment, as well as to measure electrical equipment-related parameters, provide a basis for energy metering, monitoring and protection of power lines, and thus ensure the safety of power systems. Economic Operation. Low-voltage current transformers are often used as low-voltage distribution networks to measure and protect end-users' important equipment for safe and reliable use of electricity. They are used in a large number of low-voltage power systems, and annual usage increases at a rate of $8 \%$.

In order to ensure the accuracy of energy metering, low-voltage current transformers need to be tested by a tripartite authorized agency before they are installed on site and formally put into operation. Generally, such tests are performed manually by personnel, which brings many drawbacks. Manual testing requires manual handling of transformers, manual access, demolition, switching lines, and manual operation of equipment, which consumes enormous human and material resources. Manual wiring and disconnection can easily cause electric shocks and threaten the safety of workers. Normally, only a few dozen tests can be done every day, and the efficiency is very low.With the rapid increase in the demand for low-voltage current transformers, the verification workload has also increased. The drawbacks of manual verification of current transformers have become increasingly prominent.Based on this background, some scientific research institutes and companies engaged in the verification of transformers have invested a lot of effort in developing devices capable of self-calibrating current transformers. Developing an intelligent verification system for low-voltage current transformers has important practical significance.

With the advancement of automation technology, the currently-developed transformer device can automatically complete the switching of measurement lines. Through software program control, various functional tests including pressure resistance, demagnetization, instrument security factor, magnetic saturation, and basic error are completed and automatically Generate results. However, the primary and secondary wiring of transformers must also be manually completed, which is a large amount of work.

The automatic wiring and dis-wiring is the core technology for metering transformer automatic testing process, which will greatly save labor and increase testing efficiency. The realization of 
automatic wiring and disconnection in the popularization of mechatronic technology is not a problem today. However, it is not easy to ensure that each wiring is accurate, high success rate and quite stable. Due to the different quality control capabilities of the manufacturer, there is a certain deviation between the size of the actual transformer produced and the standard size, such as the external dimensions, the position of the secondary screw, the relative size of the center of the base and the center of the main body. When the transformer enters the verification station, the actual small deviation will affect the contact area of the secondary crimp, and it is difficult to ensure $100 \%$ accuracy of the automation wiring.

In order to solve the problem of positional deviation of transformers and improve the success rate of automated crimping, a set of positioning and correcting device is designed in this paper, and the attitude of the transformer is corrected by animatronics control. Precise structural design and algorithmic logic control can correct minor dimensional deviations, improve the problem of ineffective contact with the secondary conductor string in automated wiring, and reduce manual intervention.

\section{Mechanical structure design and working principle}

A typical low-voltage current transformer integrated test device schematic shown in Fig. 1. In this figure, multiple transformers under test have been fixed to the fixed station via a transport system, and a large-current copper wire is connected through a copper wire automatic threading mechanism. After the verification test is completed, the transformer will be returned to the loading area by the original route of the transportation line, and the feeding and packing will be completed manually.

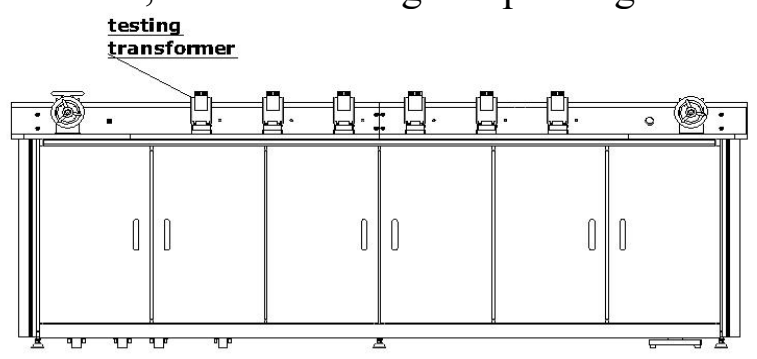

Fig. 1. The schema of integrated testing bench.

The automatic wiring structure consists of a programmable controller, a secondary wiring mechanism, a changeover mechanism, a guide mechanism, a servomotor and a proximity switch sensor. The secondary connection mechanism is pushed by the air cylinder, so that the connection terminal is crimped to the secondary terminal of the transformer, and the secondary electric circuit wiring is realized. After the transformer is fixed and a copper wire penetrates, the secondary electrical circuit will be controlled by the above-mentioned mechanism to complete the automatic wiring, so as to realize the entire process unmanned operation. The automatic wiring structure is shown in Fig. 2.

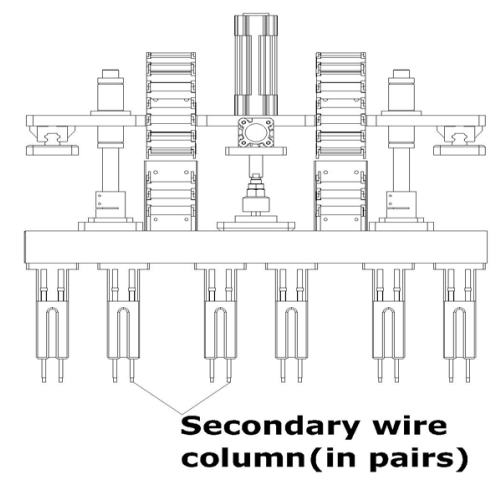

Fig. 2. The automatic wiring structure.

The positioning correction device will act on the transformer of the above-mentioned test bench body and complete the correction and fixation of the position of the test object before the connection 
device works. It includes a correcting and pushing component and a correcting and positioning clamping component. The correcting and pushing component has a fixed baffle fixed on the detection platform, and adopts a drive device to realize forward and backward movement.

The components such as jaws are extended by the electric cylinder and moved along the guide rails to the designated position. The claws are closed and the clamping jaws of the transformer are driven by the correct closing jaws. Since the rectification gripper has two symmetrical movement actuators, the transformer width centering can be realized. Push the cylinder drive out and push the movable baffle to push the transformer along the guide shaft to adjust the wiring position of the irregular transformer. If the transformer is not regular, the transformer will be pushed by the thrust and the ball slides to the regular position, which can make the transformer. The secondary terminal position is kept within \pm 0.5 of the specified range to ensure the consistency of the crimping position. The main mechanical structure of the positioning correction device is shown in Fig. 3.

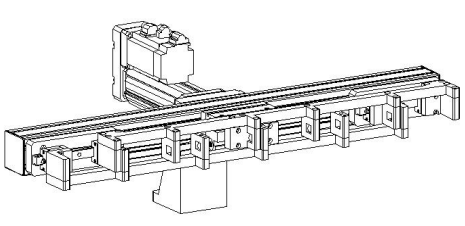

Part1: Adaptive Jaws

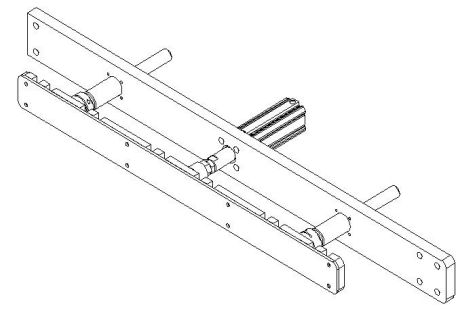

Part2: Active thrust baffle

Fig. 3. The main mechanical structure of the positioning correction device.

\section{PLC control algorithm design}

The PLC control overall algorithm flow chart is shown in Fig. 3. . In order to ensure the correct stability of the connection and the security of the device, there are many proximity switches that are configured on the system to detect actions. When the proximity switch detects the action is not in place.

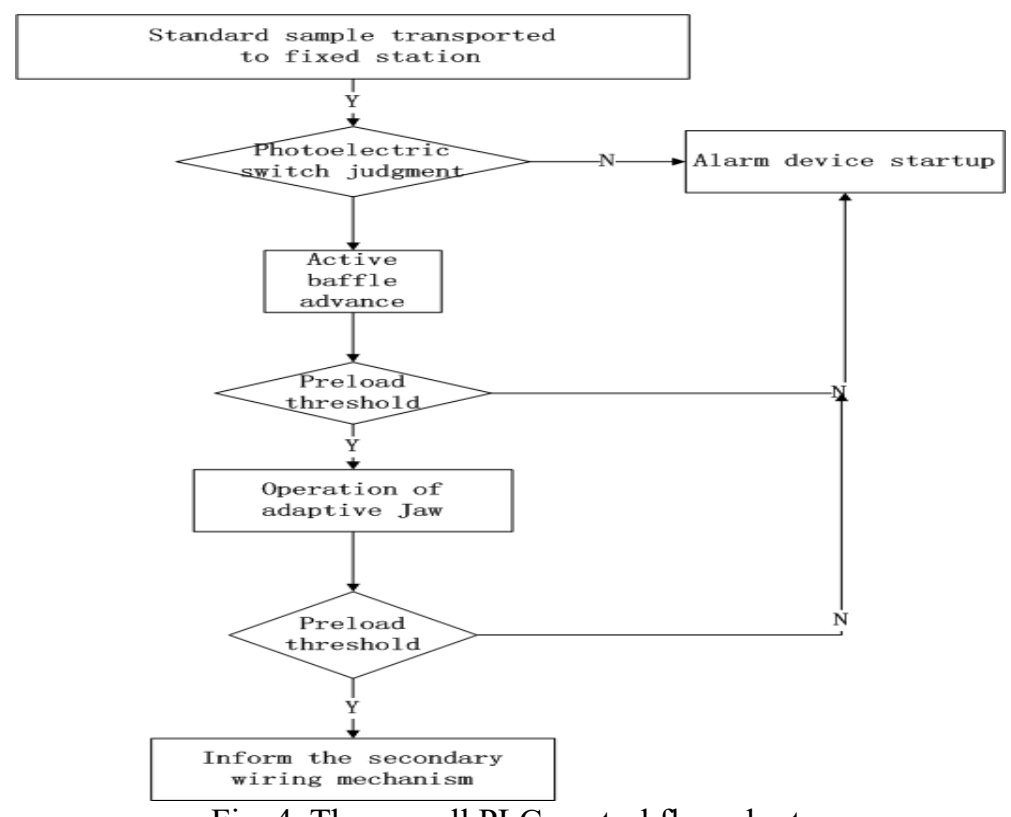

Fig. 4. The overall PLC control flow chart.

In this system, the control part adopts Siemens S7-300 series PLC, which controls the action of the on-site disconnection mechanism and can meet the automation control requirements of this kind of equipment. As the touch screen in this system requires more screens, more control points and more complex screen scripts, taking into account the aesthetic appearance of the screen and the reliability of the operation, Siemens chose the MP277 touch screen. The Siemens S7-300 is the heart of the 
control system. It uses FESTO servo-controlled motors to drive the reversing mechanism as well as through copper rods and copper bars. Because the control system requires more pneumatically driven structural components, the valve island is used in this system to drive the primary wiring structure, the secondary wiring mechanism and the guiding mechanism, and a FESTO cylinder-equipped proximity switch is used to detect whether the mechanical action is in place.

\section{Conclusions}

This article presents a positioning correction device based on PLC adaptive control and its mechanical structure design. This design is mainly composed of the corrective pushing part and the correcting and positioning clamping part. Through the joint action of the thrust and the clamping force, the position of the dimensional deviation transformer sample can be re-fixed and calibrated.The practical application results show that after the calibration, the success rate of automatic wiring is significantly increased, which effectively reduces the manual intervention in the automatic detection process, improves the detection efficiency, the detection stability, and the safety of personnel work.

\section{References}

[1] G. Deng, C. Zhong, L. Wang, H. Dai, J. Dai, and X. Yuan, Database research on automatic testing system for metering current transformers, Water Resources and Power, vol. 31 (7), pp. 33-36, 2013.

[2] Wang L H, Yao S H, and Li Y H, Risk Assessment and Risk Warning for Current Transformer Automatic Verification System, Applied Mechanics \& Materials, vol. 7, pp. 809-814, 2014.

[3] Lavie F, Pavy S, and Dernis E, Development of automatic verification system with integrated low voltage current transformer, Automation \& Instrumentation, vol. 74(4), pp. 338-345, 2013.

[4] G. Deng, X. Ma, and Z. Cao, Research and application of $0.4 \mathrm{kV}$ metering current transformers automatic verification system, Electrical Measurement \& Instrumentation, vol. 50(573), pp. 95-99, 2013.

[5] Deng Z, Wang X, and Wang L,. Research on Flexible Configuration of Auto Verification System of Low-voltage Current Transformer, Water Resources \& Power, vol. 10(4), pp. 256-260, 2013.

[6] Wang L H, Yao S H, and Li Y H, Risk Assessment and Risk Warning for Current Transformer Automatic Verification System, Applied Mechanics \& Materials, vol. 7, pp. 809-814, 2014.

[7] Cheng Z, Scanner Drive of Auto Verification System Based on Low-Voltage Current Transformer, Computer \& Digital Engineering, vol.22(9), pp. 671-675, 2013. 